\title{
Stochastic nonlinear dynamics of the cardiovascular system
}

\author{
P.V.E. McClintock ${ }^{1} \&$ A. Stefanovska ${ }^{2,1}$ \\ 'Department of Physics, Lancaster University, Lancaster, UK. \\ ${ }^{2}$ Group of Nonlinear Dynamics and Synergetics, Faculty of Electrical \\ Engineering, University of Ljubljana, Tržaška, Slovenia.
}

\begin{abstract}
The human cardiovascular system (CVS) is a highly complex mechanism. Signals derived from it are difficult to analyse because they are time-varying, noisy, and of necessarily limited duration. The application of techniques drawn from nonlinear science has, however, yielded many insights into the nature of the CVS, and has provided strong evidence for a large degree of determinism in the way it functions. Yet there is compelling evidence that random fluctuations (noise) also play an important role. The extent to which the CVS can be modelled as a stochastic nonlinear dynamical system is reviewed, and future research and possible applications based on this perception are discussed.
\end{abstract}

\section{Introduction}

The non-constancy of the human heart rate has been appreciated for a very long time [1], but the underlying causes are less than immediately obvious and have been the subject of a continuing debate. With improved signal acquisition techniques and more sophisticated methods of data analysis, however, the picture is becoming clearer. As we discuss below, it seems that the heart rate variations are largely deterministic, but with superimposed random fluctuations (noise) giving rise to changed behaviour and to a variety of interesting phenomena. Furthermore, the heart rate is not the only oscillatory process whose frequency and amplitude vary with time. The same is true for respiration, and there are also at least three other oscillatory processes of lower frequency that behave in a similar way. As we shall see, there are good reasons why the whole cardiovascular system (CVS) may 


\section{8}

Simulations in Biomedicine $V$

be considered as a single entity that can usefully be modelled as a set of interacting oscillators.

We start by describing briefly the kinds of signals that can be acquired, on which any understanding of CVS dynamics must be based. In Sec. 3 we consider the extent to which the data suggest analogies to interacting deterministic oscillators. Then, in Sec. 4 we discuss measurements and phenomena that seem to require the existence of significant stochasticity in the system, concentrating especially on synchronization phenomena. In Sec. 5 we consider the kind of model that appears to be required to account for the observations, and we review briefly some preliminary attempts to construct a digital model of this kind. Finally, in Sec. 6 we draw conclusions and try to identify promising future directions and possible applications of the research.

\section{Cardiovascular signals}

In order to study, not just the heart in isolation, but the CVS as a whole, it is desirable to make several different kinds of measurement simultaneously [2]. Fig. 1(a) shows a set of such results where ECG, blood pressure, and blood flow rate were recorded simultaneously, for a healthy subject in repose, over a period of $20 \mathrm{~min}$ utes. Interest often centres on the bloodflow circulatory control mechanisms, and so any processes occurring on timescales longer than about 1 minute (the average circulation period) are ignored. Fig. 1(b) shows the averaged wavelet transform of
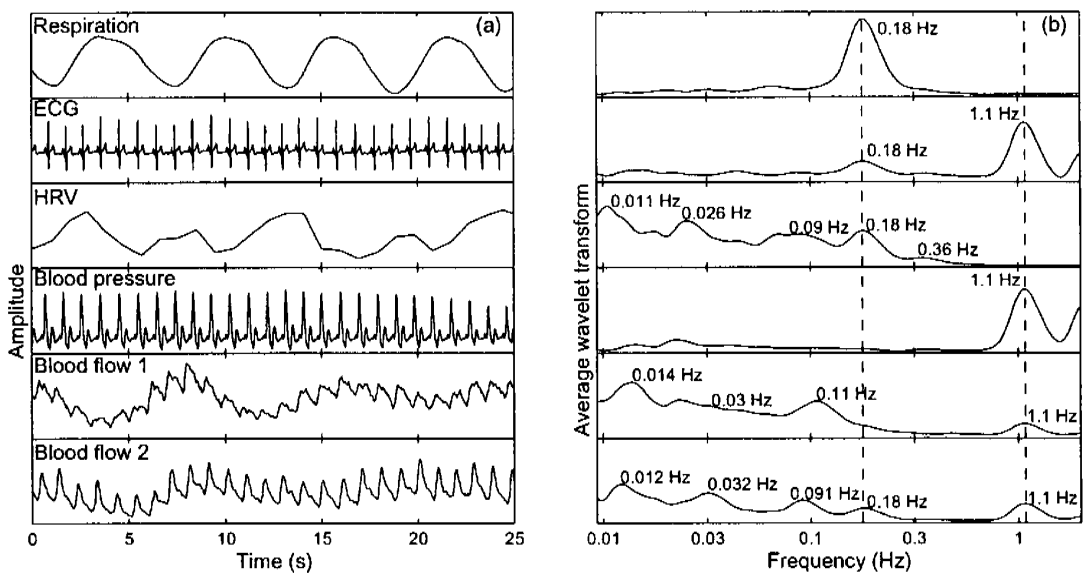

Figure 1: Samples of cardiovascular signals (a) and time averages of their wavelet transforms (b). The positions of the peaks are almost the same for all signals, while the corresponding amplitudes may be considerably different. After [2]. 
the same data, but calculated over the full 20 minutes. A detailed discussion of such results is presented in [2], but it is immediately evident that -

- There are (at least) five characteristic spectral peaks.

- Remarkably, the same, or almost the same, peaks appear in all the spectra, regardless of where or how the corresponding signals were recorded, though there are considerable differences in amplitude.

- All the peaks are broadened.

Table 1: Characteristic frequencies and their probable physiological origins.

\begin{tabular}{cc}
\hline$\sim$ Frequency $(\mathrm{Hz})$ & Physiological process \\
\hline 1.0 & Heart \\
0.3 & Respiration \\
0.1 & Myogenic \\
0.04 & Neurogenic \\
0.01 & Endothelial \\
\hline
\end{tabular}

It may be inferred that the five spectral peaks arise from five underlying oscillatory processes of some kind. Their current physiological attributions are shown in Table 1. The origins of the two higher frequencies are of course obvious: they relate to the cardiac and respiratory processes. The origins of the other three frequencies are less well established, but they appear to be derived respectively from: the intrinsic myogenic activity of smooth muscle $[3,4,5]$; autonomous nervous (neurogenic) control $[6,7]$; and endothelial $[5,8]$ processes. These latter three processes relate to control mechanisms that adjust the radii of the blood vessels, and thus are distributed throughout the whole body.

\section{Deterministic aspects of the cardiovascular system}

Although the oscillatory processes implied by the spectral peaks immediately suggest deterministic activity at those frequencies (Table 1), the widths of the peaks cannot be accounted for just in terms of the width introduced by the wavelet analysis. A clue to the origin of some of this broadening can be found by inspection of the full time-frequency wavelet spectrum, part of which is shown in Fig. 2. It can be seen that both the instantaneous amplitudes and central frequencies vary in time.

It appears that the instantaneous frequency of any given spectral peak oscillates. The modulation results from oscillations with the spectral peak next-lowest in frequency, with contributions coming from all of the other oscillatory processes too. Heart rate variability (HRV), for example, is a signal representing the variations 

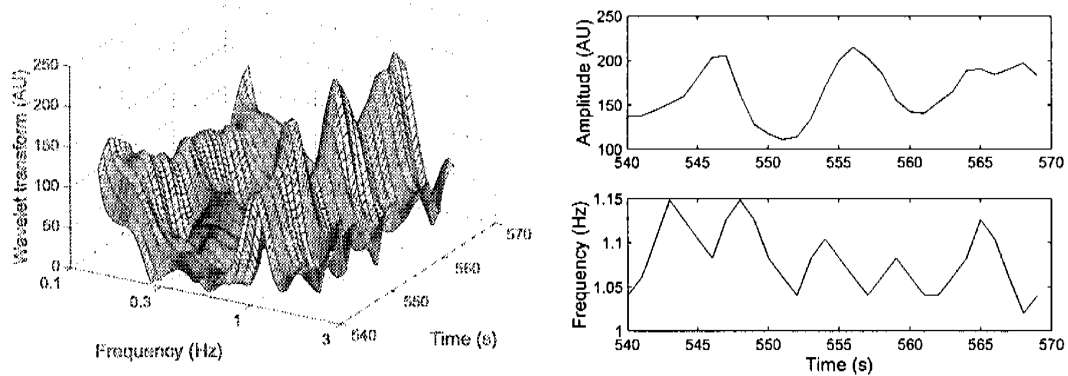

Figure 2: A segment of the wavelet transform of the blood flow signal in the timefrequency plane (left). Peaks at the heart $(\sim 1.1 \mathrm{~Hz})$ and respiration rates $(\sim 0.18 \mathrm{~Hz})$ are visible. On the right are plotted the amplitude and frequency variations of the heartrate peak. After [9].

of the cardiac frequency, and from Fig. 1(b) we can see (third spectrum from the top) that its oscillations are also modulated by the processes with frequencies near $0.011,0.026,0.10$, and $0.18 \mathrm{~Hz}$ (as well as its own second harmonic at $0.036 \mathrm{~Hz}$ ). Such observations can be construed as evidence that the five oscillatory processes are mutually coupled. They seem to influence each other via couplings at least some of which are parametric, thus giving rise to the observed frequency modulation.

The electrical activity that initiates the contraction of the heart originates at the sino-atrial node, and this periodicity has consequently been called the sinus rhythm. In adapting continuously to the needs of the body, the heart alters its rate and this alteration is known as sinus arrhythmia. Variability of the heart rate is extensively studied because of its potential for providing noninvasive measures of cardiovascular control mechanisms [10]. However, neither the physiological mechanisms of interaction, nor their dynamical properties, are fully understood.

The observed variations in the rate and the amplitude of the heart contraction can be attributed to a continuous interaction between the oscillatory processes involved in blood distribution [2]. The existence of an interaction between the cardiac and respiratory functions has been known since the 18th century when Hales carried out his celebrated experiments [1] on a horse. He found that the heart rate increased on inspiration and decreased on expiration. This frequency modulation phenomenon, known as respiratory sinus arrhythmia, has been studied extensively $[11,12,13,14,15]$ since then. It has also been recognized that the respiratory function can modify the amplitude of cardiac oscillations (stroke volume) [16]. Traube in 1865 and Hering in 1869 independently observed waves in systemic blood pressure associated [17] with respiration. Their presence in blood pressure and flow signals $[6,18,5,8]$ illustrates the propagation of the oscillations through the cardiovascular system [19]. 
The respiratory process and heart beat can be regarded as oscillators, and their mutual interaction may therefore be expected to result in an adjustment of their rhythms, sometimes leading to synchronization. Early studies [20, 21, 22, 23, $24]$ considered temporal coordination between cardiac and respiratory rates: joint interval histograms were plotted and integer ratios of frequency and distinct phase preference were analysed. An $n: 1$ synchronization of the rhythms was found in anæsthetized rabbits $[20,21]$ and in humans in various sleep stages, at rest, during physical work $[22,23,24]$, and in anæsthesia [25, 26]. It was also concluded [27] that the rhythms are generally not phase-locked, implying either that the cardiorespiratory coupling is relatively weak, or that the disruptive effects of random fluctuations (noise) are relatively strong.

\section{Stochastic aspects of the cardiovascular system}

The notion of synchronization, where the oscillations of two interacting systems become entrained with each other, was for a long time restricted to periodic oscillations. More recently, new studies of cardiorespiratory synchronization were initiated by the introduction of methods for detecting phase synchronization between non-regular or chaotic oscillators (see [28] and the references therein). In a broad sense, synchronization can be treated as the appearance of some relationship between the states of interacting systems $\mathbf{u}_{1}(t), \mathbf{u}_{2}(t)$, characterised by their phases $\Phi_{1}, \Phi_{2}$ and their generalised phase difference, $\phi_{n, m}=n \Phi_{1}-m \Phi_{2}$. A weaker condition of phase locking

$$
\left|n \Phi_{1}-m \Phi_{2}-\delta\right|<\mathrm{const},
$$

was proposed, in which case $n: m$ phase locking manifests as a variation of $\phi_{n, m}$ around a horizontal plateau. The amplitudes of phase synchronized oscillators can be quite different, and need not be related. Phase synchronization is understood as the appearance of a peak in the distribution of the cyclic relative phase

$$
\Psi_{n, m}=\phi_{n, m} \bmod 2 \pi,
$$

and interpreted as the existence of a preferred stable value of phase difference between the two oscillators.

Two methods are often used to detect instantaneous phases, $\Phi_{i}$, of the interacting oscillators from measured data: (i) marked events, and (ii) the Hilbert transform. In the first method, a repeated event that characterises one cycle of an oscillator is first identified. The phase is then usually interpolated linearly between the events, which introduces an approximation. A $2 \pi$ increase in the phase is attributed to interval between subsequent marked events. Within this interval, the instantaneous phase is

$$
\Phi(t)=2 \pi \frac{t-t_{k}}{t_{k+1}-t_{k}}+2 \pi k, t_{k} \leq t<t_{k+1}
$$

where $t_{k}$ is the time of the $k$ th marked event. Thus obtained, the phase is a monotonically increasing piecewise-linear function defined on the real line. The second method, based on the Hilbert transform, depends strongly on the quality of the 
measured signal representing the dynamics of the observed oscillator. In most living systems, measured signals are an approximation of the dynamics of the oscillatory process under observation, and are often comupted by the interfering activity of other physiological processes and noise. In both cases, therefore, phase detection involves compromise, and probably both methods should be applied before one reaches a final interpretation of a set of measurements.
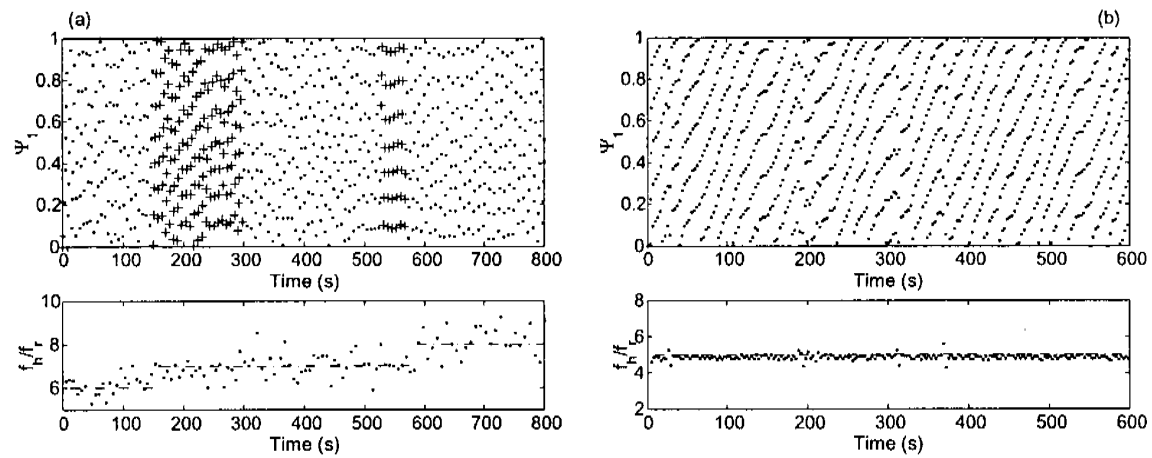

Figure 3: (a) The relative phase of heartbeats within respiratory cycles $\Psi_{1}$ and the instantaneous frequency ratio $f_{h} / f_{r}$. Between $510 \mathrm{~s}$ and $580 \mathrm{~s}$ seven horizontal lines are observed, indicating a 7:1 synchronization between the cardiac and respiratory oscillations. During this time, the generalised phase difference $\left|n \Phi_{h}-m \Phi_{r}\right|$, where $\Phi_{h}$ and $\Phi_{r}$ are the phases of heart and respiratory activities, remains constant and so also does the instantaneous frequency ratio. (b) The relative phase of heartbeats within respiratory cycles $\Psi_{1}$, and the instantaneous frequency ratio $f_{h} / f_{r}$, recorded from a critically ill subject in coma. He was heavily sedated and ventilated via a respirator at a constant frequency of $f_{r}=0.33 \mathrm{~Hz}$. His cardiac frequency is high and constant $\left(f_{h}=1.6 \mathrm{~Hz}\right)$, resulting in a constant frequency ratio, $f_{h} / f_{r}$. However, the normalised relative phase of the heartbeat within a respiratory cycle is not constant at any time of the recording. In a critical state the systems seem to be no longer capable of responding to external perturbations, including their mutual influences, so that their phases change without any relationship one to the other. From [29].

Detailed studies based on nonlinear methods, and the concepts of generalised and phase synchronization, confirmed that cardiorespiratory synchronization usually occurs in short episodes spaced at seemingly random intervals [30, 31]. Use of the synchrogram and synchronization index facilitated the detection of synchronization even where both frequencies were time-varying. The synchronization episodes in healthy subjects at rest (see Fig. 3(a)) were found to be $\sim 10$ times 

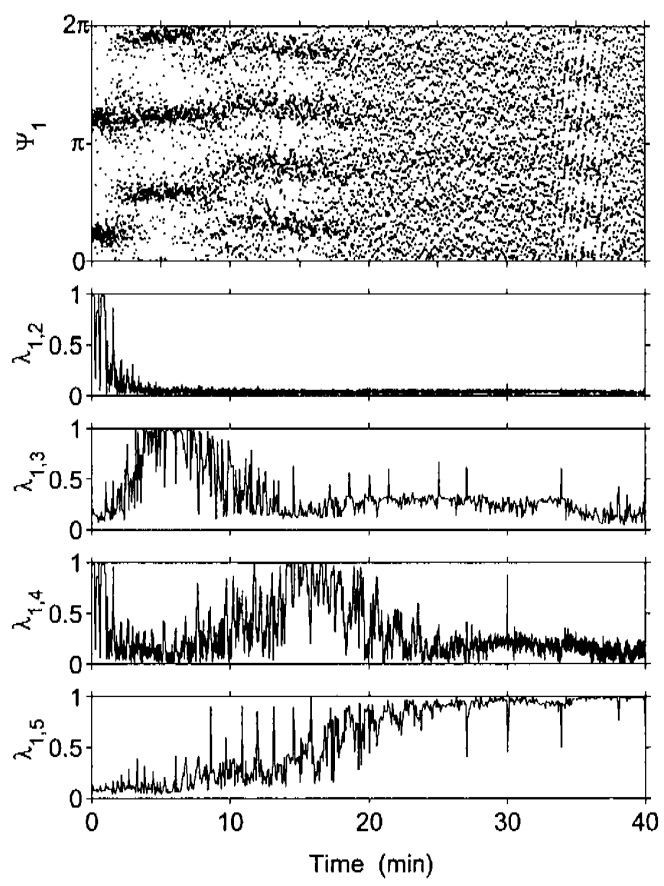

Figure 4: Synchronization phenomena in an anæsthetized rat. The anæsthetic is administered at time $=-3 \mathrm{~min}$, on the left hand side. The upper panel presents the cardiorespiratory synchrogram, showing the occurrence of transitions between 1:2,1:3,1:4 and 1:5 synchronization states as time evolves. The anæsthesia is at its deepest at $\sim 40$ minutes. The lower four panels show the synchronization indices $\lambda_{1, n}$; perfect $1: n$ synchronization is indicated by $\lambda_{1, n}=1$.

longer $(\sim 1000 \mathrm{~s})$ in athletes $[30,31]$ than in non-athletes [29]. It may reasonably be inferred that the inter-oscillator coupling strength, as revealed by the lengths of the synchronization episodes, constitutes a useful piece of information about the state of the organism. This idea was apparently confirmed by measurements on a critically ill patient in coma. As shown in Fig. 3(b), synchrogram analysis detected absolutely no sign of synchronization, even though the ratio of the cardiac and respiratory frequencies remained almost constant.

Synchronization phenomena during anaæsthesia have been studied in considerable detail [32]. Some data recorded from a rat are shown in the measurements of Fig. 4. Recording was initiated as soon as the anæsthetic had taken effect, at time $=0$ on the left hand side. Initially there is $1: 2$ synchronization, as shown by the synchrogram and by the relevant synchronization index $\lambda_{1,2}$ being close to unity. As time evolves, the anaæsthesia deepens, and the system passes through a hierarchy of different synchronization states. During deepest anaæsthesia (20-40 
minutes), the system remains locked into $1: 5$ synchronization. This process is usually reversible [32], passing back through the same set of synchronization states as the anæsthetic wears off.

Data like those shown in Fig. 4 are strongly reminiscent of earlier results obtained in lasers [33, 34], where fluctuational transitions between different modes are induced by noise, an analogy suggesting the existence of stochastic effects in the CVS. Further evidence in favour of this picture comes from recent attempts to develop models of the CVS.

\section{Modelling the cardiovascular system}

There are two compelling reasons for trying to develop a good model of the CVS. First, of course, one wishes to understand the origins of the observed phenomena, for example to account for the episodic nature of cardiorespiratory synchronisation. Secondly, by fitting the model to the measured data, one may hope to develop methods of quantifying the state of the CVS, perhaps yielding new techniques for early diagnosis of pathological conditions and for evaluation of the effects of treatment.

Any satisfactory CVS model must take account of the well-defined spectral peaks (implying the presence of oscillatory processes) and the amplitude and frequency modulation, and synchronization effects (which indicate the existence of inter-oscillator interactions), It is therefore natural to try to model the system with a set of oscillators [36] whose couplings can be adjusted [35] to try to reproduce the observed CVS behaviour.

Little experimental information exists yet about either the nature of the couplings or the details of the oscillators. So we used [36] the Poincare oscillator

$$
\begin{aligned}
& \dot{x}_{i}=-x_{i} q_{i}-\omega_{i} y_{i}+g_{x_{i}}(\mathbf{x}), \\
& \dot{y}_{i}=-y_{i} q_{i}+\omega_{i} x_{i}+g_{y_{i}}(\mathbf{y}), \quad q_{i}=\alpha_{i}\left(\sqrt{x_{i}^{2}+y_{i}^{2}}-a_{i}\right),
\end{aligned}
$$

where, $\mathbf{x}, \mathbf{y}$ are vectors of the oscillator state variables, $\alpha_{i}, a_{i}$, and $\omega_{i}$ are constants and $g_{y i}(\mathbf{y})$ and $g_{x i}(\mathbf{x})$ are linear coupling vectors. Although to some extent this choice is arbitrary, (4) possesses properties of structural stability, robustness and symmetry consistent with physiological understanding and the analyses of measured time series.

Our numerical simulation [35] of five coupled oscillators (4), with characteristic frequencies chosen to accord with measured data (Fig. 1), and amplitudes set initially to unity, generates signals that to the eye seem in many respects to resemble those from the cardiovascular system. We were especially interested to establish whether frequency and amplitude modulation would occur with short episodes of synchronization at random intervals, as observed in the experiments. The investigations are still at an early stage, but we have already established: (a) that the observed depth of modulation requires parametric couplings (as expected); (b) that with appropriately chosen parameter values parametric modulation indeed gives rise to episodes of synchronization, but of course in a totally deterministic fashion with equal intervals between the episodes; and (c) with purely linear couplings, and 

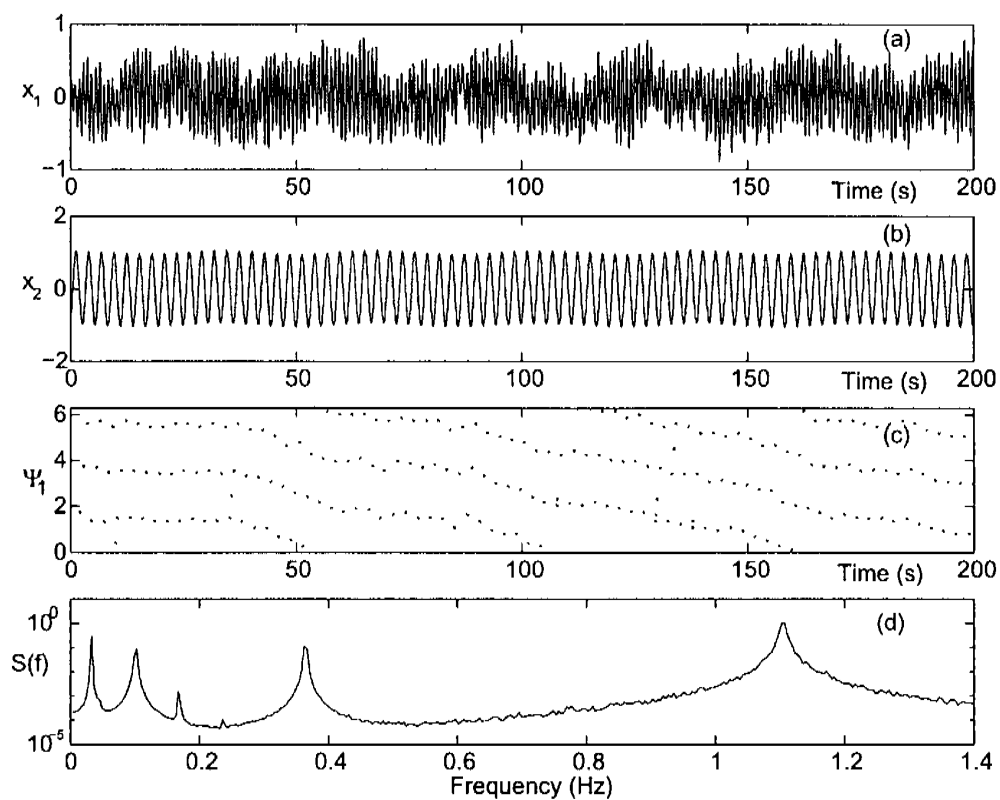

Figure 5: Results of modelling with linear couplings, in the presence of fluctuations. (a) and (b) The time series showing the rhythmic activities of the cardiac and respiratory flow components. (c) The corresponding cardiorespiratory synchrogram. (d) Power spectrum of oscillation in the blood flow generated by the cardiac activity. After [35].

added noise (random fluctuations), the synchronization episodes occur briefly and randomly just as observed in reality. An example showing the modelling of cardiorespiratory synchronization is shown in Fig. 5. It appears, therefore, that both linear and parametric couplings exist and that it is essential to take into account the influence of stochastic effects resulting from the (unmodelled) rest of the system.

\section{Conclusion and future directions}

The existence of well-defined spectral peaks and frequency and amplitude modulation phenomena observed in the CVS (Fig. 1(b)), and the fact that the system can sometimes become chaotic [37, 38], show that much CVS activity is certainly deterministic in character. On the other hand, the synchronization effects (Fig. 4) and noisy transitions between synchronization regimes provide evidence of stochasticity. It seems that the CVS should be thought of as a deterministic set of coupled oscillators, but subject to noise. Here "noise" means external random influences and all other effects not explicitly considered.

This picture is confirmed by our preliminary studies of the coupled oscillator model [36], in that it is able to reproduce [35] many features seen in the measured 
data. Comparison of the model with the data suggest that, although cardiovascular signals indeed have a strong deterministic element, random noise also plays a crucially important role. The approach we have described above relates the underlying physiological processes to particular spectral peaks and thus allows them to be studied individually. Furthermore, it promises quantitative evaluation of the couplings between them. The latter feature is potentially of particular interest for diagnosis and treatment because it would enable the function and health of the cardiovascular system as a whole to be evaluated.

\section{Acknowledgements}

It is a pleasure to acknowledge helpful discussions with Mitya Luchinsky, Maja Bračič Lotrič and Hebe Kvernmo. The work was supported by EPSRC (UK), INTAS, the Slovenian Ministry of Education, Science and Sport, and the Joy Welch Trust (UK).

\section{References}

[1] Hales, S., Statistical Essays II, Hoemastatisticks. Innings Manby: London, 1773.

[2] Stefanovska, A. \& Bračič, M., Physics of the human cardiovascular system. Contemporary Physics, 40, pp. 31-55, 1999.

[3] Johnson, P.C., The myogenic response. News Physiol Sci, 6, pp. 41-42, 1991.

[4] Bertuglia, S., Colantuoni, A. \& Intaglietta, M., Effects of L-NMMA and indomethacin on arteriolar vasomotion in skeletal-muscle microcirculation of conscious and anesthetized hamsters. Microcirc Res, 48, pp. 68-84, 1994.

[5] Kvernmo, H.D., A., S., Kirkebøen, K.A. \& Kvernebo, K., Oscillations in the human cutaneous blood perfusion signal modified by endothelium-dependent and endothelium-independent vasodilators. Microvasc Res, 57, pp. 298-309, 1999.

[6] Karstrup, J., Bühlow, J. \& Lassen, N.A., Vasomotion in human-skin before and after local heating recorded with laser doppler flowmetry - A method for induction of vasomotion. Int J Microcirc: Clin Exp, 8, pp. 205-215, 1989.

[7] Söderström, T., Stefanovska, A., Veber, M. \& Svenson, H., Involvement of sympathetic nerve activity in skin blood flow oscillations in humans. Am J Physiol: Heart Circ Physiol, in press, 2002.

[8] Stefanovska, A., Bračič, M. \& Kvernmo, H.D., Wavelet analysis of oscillations in the peripheral blood circulation measured by laser Doppler technique. IEEE Trans Bio Med Eng, 46, pp. 1230-1239, 1999.

[9] Bračič, M., McClintock, P.V.E. \& Stefanovska, A., Characteristic frequencies of the human blood distribution system. Stochastic and Chaotic Dynamics in the Lakes, eds. D.S. Broomhead, E.A. Luchinskaya, P.V.E. McClintock \& T. Mullin, American Institute of Physics: Melville, New York, pp. 146-153, 2000 . 
[10] Camm, A.J., Malik, M., Bigger, J.T. \& et al., Heart rate variability - Standards of measurement, physiological interpretation, and clinical use. Circulation, 93, pp. 1043-1065, 1996.

[11] Ludwig, C., Beiträge zur Kenntniss des Einflusses der Respirationsbewegungen auf den Blatlauf im Aortensysteme. Arch Anat Physiol und Wiss Med, 13, pp. 242-302, 1847.

[12] Anrep, G.V., Pascual, W. \& Rössler, R., Respiratory variations of the heart rate. Proc Roy Soc, London, Ser B, 119, pp. 191-230, 1936.

[13] Angelone, A. \& Coulter, N.A., Respiratory sinus arrhythmia: A frequency dependent phenomenon. $J$ Appl Physiol, 19(3), pp. 497-482, 1964.

[14] Davies, C.T.M. \& Neilson, J.M.M., Sinus arrhythmia in men at rest. $J$ Appl Physiol, 22(5), pp. 947-955, 1967.

[15] Hirsch, J.A. \& Bishop, B., Respiratory sinus arrhythmia in humans: How breathing pattern modulates heart rate. Am J Physiol: Herat Circ Physiol, 241, pp. H620-H629, 1981.

[16] Cohoon, D.H., Michael, I.E. \& Johnson, V., Respiratory modification of the cardiac output. Am J Physiol, 133, pp. 642-650, 1942.

[17] Cherniack, N.S., Heymann, M. \& Chisholm, J.C., Oscillations in blood pressure associalted with phrenic nerve activity. Nature, 214, pp. 206-207, 1967.

[18] Kvernmo, H.D., Stefanovska, A., Bračič, M., Kirkebøen, K.A. \& Kvernebo, $\mathrm{K}$, Spectral analysis of the laser Doppler perfusion signal in human skin before and after exercise. Microvasc Res, 56, pp. 173-182, 1998.

[19] Stefanovska, A. \& Hožič, M., Spatial synchronization in the human cardiovascular system. Progress of Theoretical Physics 139, pp. 270-282, 2000.

[20] Passenhofer, H. \& Kenner, T., Zur Methodik der kontinuierlichen Bestimung der Phasenbeziehung zwishen Herzshlag und Atmung. Pflügers Archiv, 355, pp. 77-83, 1975.

[21] Kenner, T., Passenhofer, H. \& Schwaberger, G., Method for the analysis of the entrainment between heart rate and ventilation rate. Pflügers Archiv, 363, pp. 263-265, 1976.

[22] Hildebrandt, G., The autonomous time structure and its reactive modifications in the human organism. Temporal Disorder in Human Oscillatory System, eds. L. Rensing, U. an der Heiden \& M.C. Mackey, Springer: Berlin, pp. 160-174, 1987.

[23] Raschke, F., Coordination in the circulatory and respiratory systems. Temporal Disorder in Human Oscillatory System, eds. L. Rensing, U. an der Heiden \& M.C. Mackey, Springer: Berlin, pp. 152-158, 1987.

[24] Raschke, F., The respiratory system - features of modulation and coordination. Rhythms in Physiological Systems, eds. H. H. \& H.P. Koepchen, Springer: Berlin, pp. 155-164, 1991.

[25] Galletly, D.C. \& Larsen, P.D., Cardioventilatory coupling during anaesthesia. Brit $J$ Ancesth, 79, pp. 35-40, 1997.

[26] Luola, P., Jäntti, V. \& Yli-Hankala, A., Respiratory sinus arrhythmia during anaesthesia: assessment of respiration related beat-to-beat heart rate variability analysis methods. Int J Clin Monit Comp, 14, pp. 241-249, 1997. 
[27] Makey, M.C. \& Glass, L., From clocks to Chaos: The Rhythms of Life. Princeton University Press: Princeton, 1988.

[28] Pikovsky, A., Rosenblum, M. \& Kurths, J., Synchronization - A Universal Concept in Nonlinear Sciences. Cambridge University Press: Cambridge, 2001.

[29] Bračič Lotrič, M., Stefanovska, A., Štajer, D. \& Urbančič-Rovan, V., Spectral components of heart rate variability determined by wavelet analysis. Physiol Meas, 21, pp. 441-457, 2000.

[30] Schäfer, C., Rosenblum, M.G. \& Kurths, J., Heartbeat synchronised with respiration. Nature, 392, pp. 239-240, 1998.

[31] Schäfer, C., Rosenblum, M.G. \& Kurths, J., Synchronization in the human cardiorespiratory system. Phys Rev E, 60, pp. 857-870, 1999.

[32] Stefanovska, A., Haken, H., McClintock, P.V.E., Hožič, M., Bajrović, F. \& Ribarič, S., Reversible transitions between synchronization states of the cardio-respiratory system. Phys Rev Lett, 85, pp. 4831-4834, 2000.

[33] Haken, H., Laser Theory. Springer: Berlin, 1970.

[34] Haken, H., Synergetics, An Introduction. Springer: Berlin, 1983.

[35] Stefanovska, A., Luchinsky, D.G. \& McClintock, P.V.E., Modelling couplings among the oscillators of the cardiovascular system. Physiol Meas, 22, pp. 551-564, 2001.

[36] Stefanovska, A., Bračič, M., Strle, S. \& Haken, H., The cardiovascular system as coupled oscillators? Physiol Meas, 22, pp. 535-550, 2001.

[37] Babloyantz, A. \& Destexhe, A., Is the normal heart a periodic oscillator? Biol Cybern, 58, pp. 203-211, 1988.

[38] Poon, C.S. \& Merrill, C.K., Decrease of cardiac chaos in congestive heart failure. Nature, 389, pp. 492-495, 1997. 